\title{
Low complexity concurrent constant modulus algorithm and soft decision directed scheme for blind equalisation
}

\author{
S. Chen
}

\begin{abstract}
The recently introduced concurrent constant modulus algorithm (CMA) and decision-directed (DD) scheme provides a state-of-the-art low-complexity blind equalisation technique for high-order quadrature amplitude modulation (QAM) channels. At a small cost of slightly more than doubling the complexity of the standard CMA blind equaliser, this concurrent CMA and DD blind equaliser achieves a dramatic improvement in equalisation performance over the CMA. In the paper, a new blind equalisation scheme is proposed based on concurrent CMA and a novel soft decision-directed (SDD) adaptation. The proposed concurrent CMA and SDD blind equaliser has simpler computational requirements than the concurrent CMA and DD algorithm. Extensive simulation shows that it has the same steadystate equalisation performance as the concurrent CMA and DD algorithm and a faster convergence speed over the latter scheme.
\end{abstract}

\section{Introduction}

Blind equalisation improves system bandwidth efficiency by avoiding the use of a training sequence. Furthermore, for certain communication systems, training is infeasible and a blind equaliser provides a practical means for combating the detrimental effects of channel dispersion in such systems. For systems employing high bandwidth-efficiency quadrature amplitude modulation (QAM) signalling, the constant modulus algorithm (CMA) based equaliser [1-4] is by far the most popular low-complexity blind equalisation scheme, as it can readily meet the real-time computational constraint. The CMA is also very robust to imperfect carrier recovery. There have been many studies which investigate the convergence behaviour of the CMA, and an excellent review can be found in [5]. A particular problem of the CMA is that its steady-state mean square error (MSE) may not be sufficiently low for the system to obtain adequate performance. A possible solution is to switch to a decision-directed (DD) adaptation which should be able to minimise the residual CMA steady-state MSE [6]. However, as pointed out in [7], in order for such a transfer to be successful, the CMA steady-state MSE should be sufficiently low. In practice, such a low level of MSE may not always be achievable by the CMA scheme.

Recently, De Castro and co-workers [7] have suggested an interesting solution to this problem. Rather than switching to a DD adaptation after the CMA has converged, they have proposed to operate a DD equaliser concurrently with a CMA equaliser. The weight adaptation of the DD equaliser follows that of the CMA equaliser and, to avoid

\footnotetext{
(C) IEE, 2003
}

IEE Proceedings online no. 20030619

doi: 10.1049/ip-vis:20030619

Paper first received 4th July 2002 and in revised form 10th April 2003

The author is with the School of Electronics and Computer Science, University of Southampton, Southampton SO17 1BJ, UK error propagation due to incorrect decisions, the DD adjustment only takes place if the CMA adaptation is judged to have achieved a successful adjustment with high probability. At a small cost of slightly more than doubling the complexity of the very simple CMA, this concurrent CMA and DD equaliser is reported to obtain a dramatic improvement in equalisation performance over the CMA [7]. Among various low-complexity blind equalisation schemes for high-order QAM channels (e.g. [8-11]), this concurrent CMA and DD scheme represents a state-of-theart technique. Another blind equalisation scheme, which is relevant to the proposed concurrent CMA and soft decisiondirected (SDD) blind equaliser, is the multi-stage blind clustering or a bootstrap maximum a posteriori probability (MAP) blind equaliser [12, 13].

The bootstrap MAP blind scheme was originally derived in [14] for the 4-QAM case and extended to $M$-QAM $(M>4)$ channels in $[12,13]$. It is only slightly more complex than the CMA in terms of computational requirements per weight update, and has been shown to outperform the concurrent CMA and DD scheme, both in terms of convergence rate and steady-state equalisation performance $[15,16]$. A drawback of this bootstrap MAP blind equaliser is that its adaptive process requires $L$-stage switchings, where $L=\log _{2}(M) / 2$, and each stage of adaptation needs a different set of algorithm parameters. Thus, tuning of the bootstrap MAP algorithm is quite complicated. The proposed concurrent CMA and SDD scheme may be viewed as operating a CMA equaliser and a last-stage bootstrap MAP equaliser concurrently. Unlike the bootstrap MAP algorithm, this concurrent CMA and SDD blind equaliser does not require complicated algorithm tuning and switching, at a cost of a small increase in complexity per weight update. The proposed concurrent CMA and SDD algorithm, however, is computationally simpler than the concurrent CMA and DD scheme. Simulation results included demonstrate that both the concurrent CMA and DD scheme and the concurrent CMA and SDD scheme have the same steady-state 
equalisation performance, but the latter has a faster convergence speed.

\section{Low-complexity blind equalisation}

Consider the baseband model of a digital communication channel in which the received signal at symbol-spaced sample $k$ is given by

$$
r(k)=\sum_{i=0}^{n-1} a_{i} s(k-i)+e(k)
$$

where $n$ is the length of the channel impulse response (CIR), $a_{i}=a_{i R}+j a_{i I}$ are the complex-valued channel tap weights, $e(k)=e_{R}(k)+j e_{I}(k)$ is a complex-valued Gaussian white noise with $\mathrm{E}\left[e_{R}^{2}(k)\right]=\mathrm{E}\left[e_{I}^{2}(k)\right]=\sigma_{e}^{2}$ and $E[\cdot]$ denoting the expectation operator, and the complex-valued symbol sequence $s(k)=s_{R}(k)+j s_{I}(k)$ is assumed to be independently identically distributed and takes the value from the $M$-QAM symbol set defined by

$$
\mathcal{S}=\left\{s_{i l}=(2 i-Q-1)+j(2 l-Q-1), \quad 1 \leq i, l \leq Q\right\}
$$

with $Q=\sqrt{M}=2^{L}$ and $L$ being an integer. To remove the channel distortion, a symbol-spaced equaliser of the form

$$
y(k)=\sum_{i=0}^{m-1} w_{i} r(k-i)=\boldsymbol{w}^{\mathrm{T}} \boldsymbol{r}(k)
$$

is employed, where $m$ is the order of the equaliser, $\boldsymbol{w}(k)=$ $\left[w_{0} w_{1} \cdots w_{m-1}\right]^{\mathrm{T}}$ is the equaliser weight vector with $w_{i}=$ $w_{i R}+j w_{i I}$, and $\boldsymbol{r}(k)=[r(k) r(k-1) \cdots r(k-m+1)]^{\mathrm{T}}$ is the equaliser input vector. To deal with non-minimumphase channels, the equaliser should have a delay of $k_{d} \approx$ $m / 2$. Before blind adaptation, the equaliser weights are initialised to $w_{i}=1+j 0$ for $i=k_{d}$ and $w_{i}=0+j 0$ for $i \neq k_{d}$. It is recognised that a fractionally spaced equaliser can often achieve better performance. The purpose of this study is to assess a group of blind equalisers under a common framework. All the blind adaptive schemes studied here are equally applicable to fractionally spaced equalisers (e.g. $[5,7,16])$.

\subsection{The constant modulus algorithm}

The CMA adjusts the equaliser weights by minimising the nonconvex cost function

$$
\bar{J}_{\mathrm{CMA}}(\boldsymbol{w})=\mathrm{E}\left[\left(|y(k)|^{2}-\Delta_{2}\right)^{2}\right]
$$

using a stochastic gradient algorithm, where $\Delta_{2}$ is a real positive constant defined by

$$
\Delta_{2}=\mathrm{E}\left[|s(k)|^{4}\right] / \mathrm{E}\left[|s(k)|^{2}\right]
$$

At sample $k$, given $y(k)=\boldsymbol{w}^{\mathrm{T}}(k) \boldsymbol{r}(k)$, the CMA adapts $\boldsymbol{w}$ according to $[1,2]$ :

$$
\left.\begin{array}{l}
\epsilon(k)=y(k)\left(\Delta_{2}-|y(k)|^{2}\right) \\
\boldsymbol{w}(k+1)=\boldsymbol{w}(k)+\mu \epsilon(k) \mathbf{r}^{*}(k)
\end{array}\right\}
$$

where $\mu$ is a small positive adaptive gain and $\boldsymbol{r}^{*}(k)$ is the complex conjugate of $\boldsymbol{r}(k)$. The CMA has a very simple computational complexity, as summarised in Table 1. Although $M$-QAM symbols do not fall on the circle of radius $\sqrt{\Delta_{2}}$, it is known that the cost function $\bar{J}_{\mathrm{CMA}}(\boldsymbol{w})$ is minimised at the equaliser weight solution which restores
Table 1: Comparison of computational complexity per weight update

\begin{tabular}{llll}
\hline Equaliser & Multiplications & Additions & $\begin{array}{l}\exp (\cdot) \\
\text { evaluations }\end{array}$ \\
\hline CMA & $8 \times m+6$ & $8 \times m$ & - \\
CMA + DD & $16 \times m+8$ & $20 \times m$ & - \\
CMA + SDD & $12 \times m+29$ & $14 \times m+21$ & 4 \\
\hline
\end{tabular}

Equaliser order is $m$

the signal constellation. Under certain conditions, the CMA converges to this solution subject to a possible phase shift. The CMA is known to be very robust and is capable of opening an 'initially closed eye'. The standard results on convergence analysis of the CMA have been summarised in [5]. Typically, a very small adaptive gain $\mu$ has to be used to ensure convergence.

\subsection{The concurrent CMA and decision directed equaliser}

De Castro and co-workers [7] proposed a blind equalisation scheme that consists of a CMA equaliser and a DD equaliser operating concurrently. Specifically, let

$$
\boldsymbol{w}=\boldsymbol{w}_{c}+\boldsymbol{w}_{d}
$$

Here, $\boldsymbol{w}_{c}$ is the weight vector of the CMA equaliser which is designed to minimise the CMA cost function $\bar{J}_{\mathrm{CMA}}(\boldsymbol{w})$ by adapting $\boldsymbol{w}_{c}$, and $\boldsymbol{w}_{d}$ is the weight vector of the DD equaliser which is designed to minimise the decision based MSE:

$$
\bar{J}_{\mathrm{DD}}(\boldsymbol{w})=\frac{1}{2} \mathrm{E}\left[|\mathcal{Q}[y(k)]-y(k)|^{2}\right]
$$

by adjusting $\boldsymbol{w}_{d}$, where $\mathcal{Q}[y(k)]$ denotes the quantised equaliser output defined by

$$
\mathcal{Q}[y(k)]=\underset{s_{i l} \in S}{\arg \min }\left|y(k)-s_{i l}\right|^{2}
$$

More precisely, at sample $k$, given

$$
y(k)=\boldsymbol{w}_{c}^{\mathrm{T}}(k) \boldsymbol{r}(k)+\boldsymbol{w}_{d}^{\mathrm{T}}(k) \boldsymbol{r}(k)
$$

the CMA part adapts $\boldsymbol{w}_{c}$ according to the rule (6) by substituting $\boldsymbol{w}_{c}$ in the place of $\boldsymbol{w}$ with an adaptive gain $\mu_{c}$. The DD adaptation follows immediately after the CMA adaptation but it only takes place if the CMA adjustment is viewed to be a successful one. Let

$$
\tilde{y}(k)=\boldsymbol{w}_{c}^{\mathrm{T}}(k+1) \boldsymbol{r}(k)+\boldsymbol{w}_{d}^{\mathrm{T}}(k) \boldsymbol{r}(k)
$$

Then the DD part adjusts $\boldsymbol{w}_{d}$ according to [7]:

$$
\begin{aligned}
\boldsymbol{w}_{d}(k+1)= & \boldsymbol{w}_{d}(k)+\mu_{d} \delta(\mathcal{Q}[\tilde{y}(k)]-\mathcal{Q}[y(k)]) \\
& \times(\mathcal{Q}[y(k)]-y(k)) \boldsymbol{r}^{*}(k)
\end{aligned}
$$

where $\mu_{d}$ is the adaptive gain of the DD equaliser and the indicator function

$$
\delta(x)=\left\{\begin{array}{l}
1, x=0+j 0 \\
0, x \neq 0+j 0
\end{array}\right.
$$

It can be seen that $\boldsymbol{w}_{d}$ is updated only if the equaliser hard decisions before and after the CMA adaptation are the same. 
A potential problem of (hard) decision-directed adaptation is that, if the decision is wrong, error propagation occurs which subsequently degrades equaliser adaptation. As analysed in [7], if the equaliser hard decisions before and after the CMA adaptation are the same, the decision probably is correct. The decision-directed adaptation, when it is safe to perform, has a much faster convergence speed and is capable of lowering the steady-state MSE, compared with the pure CMA. The complexity of this concurrent CMA and DD blind equaliser, summarised in Table 1, is still linear in the equaliser order $m$. Obviously, this concurrent CMA and DD blind equaliser combines the advantages of both the CMA and decision-directed adaptation. It does not suffer from a serious phase shift problem as the CMA does, and automatically performs a gain control or scaling for the restoration of the signal constellation (2). The adaptive gain $\mu_{d}$ for the DD equaliser can often be chosen much larger than $\mu_{c}$ for the CMA. Nevertheless, care must be exercised in choosing $\mu_{d}$ to avoid setting it to too high a value, which would cause serious error propagation due to incorrect decisions.

\subsection{The concurrent CMA and soft decision directed equaliser}

Denote the CIR as $\boldsymbol{a}=\left[a_{0} a_{1} \cdots a_{n-1}\right]^{\mathrm{T}}$. Then the combined impulse response of the channel and equaliser is given by

$$
\boldsymbol{f}=\left[f_{0} f_{1} \cdots f_{n_{c}-1}\right]^{\mathrm{T}}=\boldsymbol{a} \star \boldsymbol{w}
$$

where $\star$ denotes the convolution and $n_{c}=n+m-1$ is the length of the combined impulse response. Let the main tap of $\boldsymbol{f}$ be $f_{k_{d}}$. Assume that an automatic gain control is performed so that $f_{k_{d}}=1$. Note that, for the DD adaptation discussed in the previous subsection and the SDD adaptation presented in this subsection, this is automatically achieved. The equaliser soft output $y(k)$ is obviously given by

$$
y(k)=s\left(k-k_{d}\right)+\sum_{\substack{i=0 \\ i \neq k_{d}}}^{n_{c}-1} f_{i} s(k-i)+\sum_{i=0}^{m-1} w_{i} e(k-i)
$$

Therefore, after the equalisation is accomplished, the equaliser output can be expressed approximately in two terms:

$$
y(k) \approx x(k)+v(k)
$$

where $x(k)=s\left(k-k_{d}\right), k_{d}$ is an integer, and $v(k)=$ $v_{R}(k)+j v_{I}(k)$ is approximately a Gaussian white noise (e.g. [17]). Thus, if the equaliser weights have been chosen correctly, the equaliser output can be modelled approximately by $M$ Gaussian clusters with the cluster means being $s_{i l}$ for $1 \leq i, l \leq Q$. All the clusters have an approximate covariance,

$$
\left[\begin{array}{cc}
\mathrm{E}\left[v_{R}^{2}(k)\right] & \mathrm{E}\left[v_{R}(k) v_{I}(k)\right] \\
\mathrm{E}\left[v_{I}(k) v_{R}(k)\right] & \mathrm{E}\left[v_{I}^{2}(k)\right]
\end{array}\right] \approx\left[\begin{array}{cc}
\rho & 0 \\
0 & \rho
\end{array}\right]
$$

Under the above conditions, the a posteriori probability density function (p.d.f.) of $y(k)$ is

$$
p(\boldsymbol{w}, y(k)) \approx \sum_{q=1}^{Q} \sum_{l=1}^{Q} \frac{p_{q l}}{2 \pi \rho} \exp \left(-\frac{\left|y(k)-s_{q l}\right|^{2}}{2 \rho}\right)
$$

where $p_{q l}$ are the a priori probabilities of $s_{q l}, 1 \leq q, l \leq Q$, and they are all equal.

The computation of the p.d.f. (18) involves the evaluation of $M \exp (\cdot)$ function values. A local approximation can be

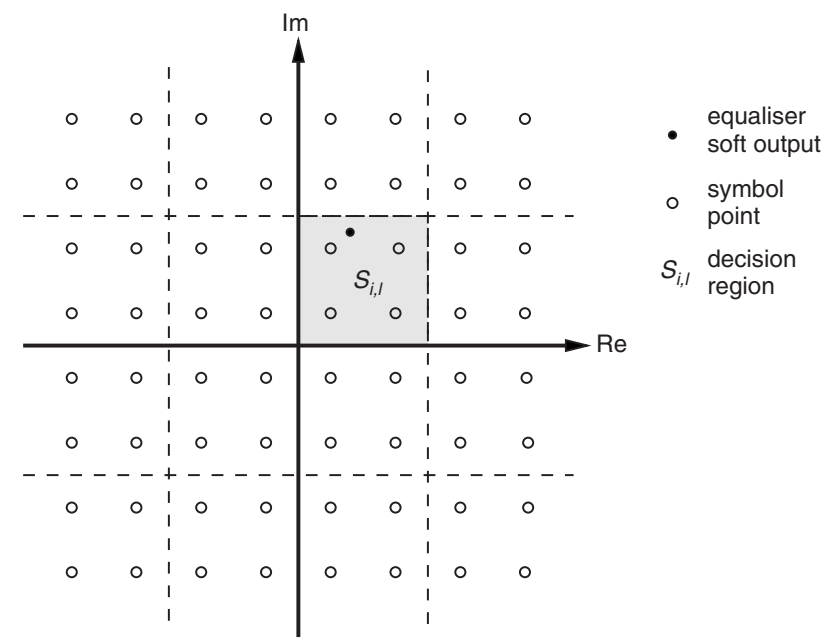

Fig. 1 Local decision regions for soft decision-directed adaptation with 64QAM constellation

adopted for this p.d.f., which only evaluates four $\exp (\cdot)$ function values. This is achieved by dividing the complex plane into $M / 4$ regular regions, as illustrated in Fig. 1. Each region $S_{i, l}$ contains four symbol points:

$$
S_{i, l}=\left\{s_{p q}, p=2 i-1,2 i, q=2 l-1,2 l\right\}
$$

If the equaliser output $y(k)$ is within the region $S_{i, l}$, a local approximation to the a posteriori p.d.f. of $y(k)$ is

$$
\hat{p}(\boldsymbol{w}, y(k)) \approx \sum_{p=2 i-1}^{2 i} \sum_{q=2 l-1}^{2 l} \frac{1}{8 \pi \rho} \exp \left(-\frac{\left|y(k)-s_{p q}\right|^{2}}{2 \rho}\right)
$$

where each a priori probability has been set to $\frac{1}{4}$. Obviously this approximation is only valid when the equalisation goal has been accomplished. A bootstrap optimisation process, however, can be performed to achieve the MAP solution, as is presented in $[12,13]$.

The proposed scheme operates a CMA equaliser and a SDD equaliser concurrently. The CMA part is identical to that of the concurrent CMA and DD scheme. The purpose of this CMA sub-equaliser is to open the eye, so that the local p.d.f. expression (20) is approximately valid. The SDD equaliser is designed to maximise the $\log$ of the local a posteriori p.d.f. criterion

$$
\bar{J}_{\text {LMAP }}(\boldsymbol{w})=\mathrm{E}\left[J_{\text {LMAP }}(\boldsymbol{w}, y(k))\right]
$$

by adjusting $\boldsymbol{w}_{d}$ using a stochastic gradient algorithm, where

$$
J_{\text {LMAP }}(\boldsymbol{w}, y(k))=\rho \log (\hat{p}(\boldsymbol{w}, y(k)))
$$

A rationale behind the maximisation of the a posteriori p.d.f. of the equaliser output $y(k)$ is that the local maxima of this p.d.f. occur at valid source symbols $[12,13]$. Hence, it seems reasonable to modify the equaliser weights so that its output is driven to these maxima. Specifically, the SDD equaliser adapts $\boldsymbol{w}_{d}$ according to

$$
\boldsymbol{w}_{d}(k+1)=\boldsymbol{w}_{d}(k)+\mu_{d} \frac{\partial J_{\mathrm{LMAP}}(\boldsymbol{w}(k), y(k))}{\partial \boldsymbol{w}_{d}}
$$

where 


$$
\begin{aligned}
& \frac{\partial J_{\text {LMAP }}(\boldsymbol{w}, y(k))}{\partial \boldsymbol{w}_{d}} \\
= & \frac{\sum_{p=2 i-1}^{2 i} \sum_{q=2 l-1}^{2 l} \exp \left(-\frac{\left|y(k)-s_{p q}\right|^{2}}{2 \rho}\right)\left(s_{p q}-y(k)\right)}{\sum_{p=2 i-1}^{2 i} \sum_{q=2 l-1}^{2 l} \exp \left(-\frac{\left|y(k)-s_{p q}\right|^{2}}{2 \rho}\right)} \boldsymbol{r}^{*}(k)
\end{aligned}
$$

and $\mu_{d}$ is an adaptive gain. The choice of $\rho$ should ensure a proper separation of the four clusters in $S_{i, l}$. If the value of $\rho$ is too large, a desired degree of separation may not be achieved. On the other hand, if too small a $\rho$ is used, the algorithm attempts to impose a very tight control in the size of clusters and may fail to do so. Apart from these two extreme cases, the performance of the algorithm does not critically depend on the value of $\rho$. As the minimum distance between the two neighbouring symbol points is 2 , typically $\rho$ is chosen to be $<1$.

Note that the operations of the CMA and SDD parts occur in a truly concurrent manner, i.e. they are both based on $y(k)$ and there is no need to compute $\tilde{y}(k)$ of (11) as the CMA and DD scheme does. The soft decision nature is evident in (24). Rather than being committed to a single hard decision $\mathcal{Q}[y(k)]$ as the DD scheme is, alternative decisions are also considered in a local region $S_{i, l}$ that includes $\mathcal{Q}[y(k)]$, and each tentative decision is weighted by an exponential term $\exp (\cdot)$ which is a function of the distance between the equaliser soft output $y(k)$ and the tentative decision $s_{p q}$. This soft decision nature enables a simultaneous update of $\boldsymbol{w}_{c}$ and $\boldsymbol{w}_{d}$ without worrying about error propagation and, therefore, simplifies the operation. This has an effect that a larger adaptive gain $\mu_{d}$ can often be used, compared with the DD scheme. It is also obvious that this SDD scheme corresponds to the last stage of the bootstrap MAP scheme given in $[12,13]$. The complexity of this concurrent CMA and SDD scheme is compared with those of the pure CMA scheme and the concurrent CMA and DD scheme of [7] in Table 1. The four $\exp (\cdot)$ evaluations can be implemented through a look-up table in practice. It can be seen that the computational complexity per weight update of this proposed new scheme is simpler than that of the concurrent CMA and DD scheme.

\section{Simulation study}

The performance of the proposed low-complexity blind equaliser was compared with that of the concurrent CMA and DD one in a computer simulation using the standard CMA blind equaliser as a benchmark. Two performance criteria were used to assess the convergence rate of a blind equaliser. The first one was an estimated MSE at each adaptation sample based on a block of $N_{\text {MSE }}$ data samples. The second one was the maximum distortion (MD) measure defined by
Table 2: 22-tap channel impulse response from [18]

\begin{tabular}{lrrrrr}
\hline $\begin{array}{l}\text { Tap } \\
\text { no. }\end{array}$ & Real & Imaginary & $\begin{array}{l}\text { Tap } \\
\text { no. }\end{array}$ & Real & Imaginary \\
\hline 0 & 0.0145 & -0.0006 & 11 & 0.0294 & -0.0049 \\
1 & 0.0750 & 0.0176 & 12 & -0.0181 & 0.0032 \\
2 & 0.3951 & 0.0033 & 13 & 0.0091 & 0.0003 \\
3 & 0.7491 & -0.1718 & 14 & -0.0038 & -0.0023 \\
4 & 0.1951 & 0.0972 & 15 & 0.0019 & 0.0027 \\
5 & -0.2856 & 0.1896 & 16 & -0.0018 & -0.0014 \\
6 & 0.0575 & -0.2096 & 17 & 0.0006 & 0.0003 \\
7 & 0.0655 & 0.1139 & 18 & 0.0005 & 0.0000 \\
8 & -0.0825 & -0.0424 & 19 & -0.0008 & -0.0001 \\
9 & 0.0623 & 0.0085 & 20 & 0.0000 & -0.0002 \\
10 & -0.0438 & 0.0034 & 21 & 0.0001 & 0.0006 \\
\hline
\end{tabular}

Table 3: 5-tap channel impulse response

\begin{tabular}{lcc}
\hline Tap no. & Real & Imaginary \\
\hline 0 & -0.2 & 0.3 \\
1 & -0.5 & 0.4 \\
2 & 0.7 & -0.6 \\
3 & 0.4 & 0.3 \\
4 & 0.2 & 0.1 \\
\hline
\end{tabular}

$$
M D=\frac{\sum_{i=0}^{n_{c}-1}\left|f_{i}\right|-\left|f_{i_{\max }}\right|}{\left|f_{i_{\max }}\right|}
$$

with

$$
f_{i_{\max }}=\max \left\{f_{i}, 0 \leq i \leq n_{c}-1\right\}
$$

The equaliser output signal constellation after convergence was also shown using $N_{\text {test }}=6000$ testing data samples not used in adaptation.

The equaliser order $m$ should be chosen sufficiently long to ensure the capability of opening closed eye and good steady-state performance, but not too long, which can cause the problems of seriously enhancing noise and slow convergence rate. The actual value of $m$ used in the simulation was found empirically. The adaptive gain $\mu$ for the CMA, the two adaptive gains $\mu_{c}$ and $\mu_{d}$ for the CMA and DD algorithm, and the adaptive gains $\mu_{c}$ and $\mu_{d}$ and the width $\rho$ for the CMA and SDD algorithm were also chosen empirically to ensure fast convergence speed and good steady-state performance. Two channels were used in evaluation, and their CIRs are given in Tables 2 and 3, respectively. In all the cases, a 23-tap equaliser was adopted. Table 4 lists the algorithm parameters used in the simulation for the three blind

\begin{tabular}{|c|c|c|c|c|c|c|c|}
\hline \multirow[t]{2}{*}{ Example } & \multirow[t]{2}{*}{$N_{M S E}$} & \multirow{2}{*}{$\begin{array}{l}\text { CMA } \\
\mu\end{array}$} & \multicolumn{2}{|l|}{$\mathrm{CMA}+\mathrm{DD}$} & \multicolumn{3}{|c|}{$\mathrm{CMA}+\mathrm{SDD}$} \\
\hline & & & $\mu_{c}$ & $\mu_{d}$ & $\mu_{c}$ & $\mu_{d}$ & $\rho$ \\
\hline 1 & 250 & $10^{-5}$ & $10^{-5}$ & 0.0001 & $10^{-5}$ & 0.001 & 0.6 \\
\hline 2 & 500 & $5 \times 10^{-7}$ & $5 \times 10^{-7}$ & $5 \times 10^{-5}$ & $5 \times 10^{-7}$ & 0.0002 & 0.6 \\
\hline 3 & 1000 & $2 \times 10^{-8}$ & $2 \times 10^{-8}$ & $5 \times 10^{-5}$ & $2 \times 10^{-8}$ & $5 \times 10^{-5}$ & 0.7 \\
\hline 4 & 1000 & $10^{-8}$ & $10^{-8}$ & $2 \times 10^{-5}$ & $10^{-8}$ & $3 \times 10^{-5}$ & 0.8 \\
\hline
\end{tabular}
equalisers ( $\mu$ for the CMA, $\mu_{c}$ and $\mu_{d}$ for the $\mathrm{CMA}+\mathrm{DD}$, and $\mu_{c}, \mu_{d}$ and $\rho$ for the $\left.\mathrm{CMA}+\mathrm{SDD}\right)$,

Table 4: Algorithm parameter settings in simulation 

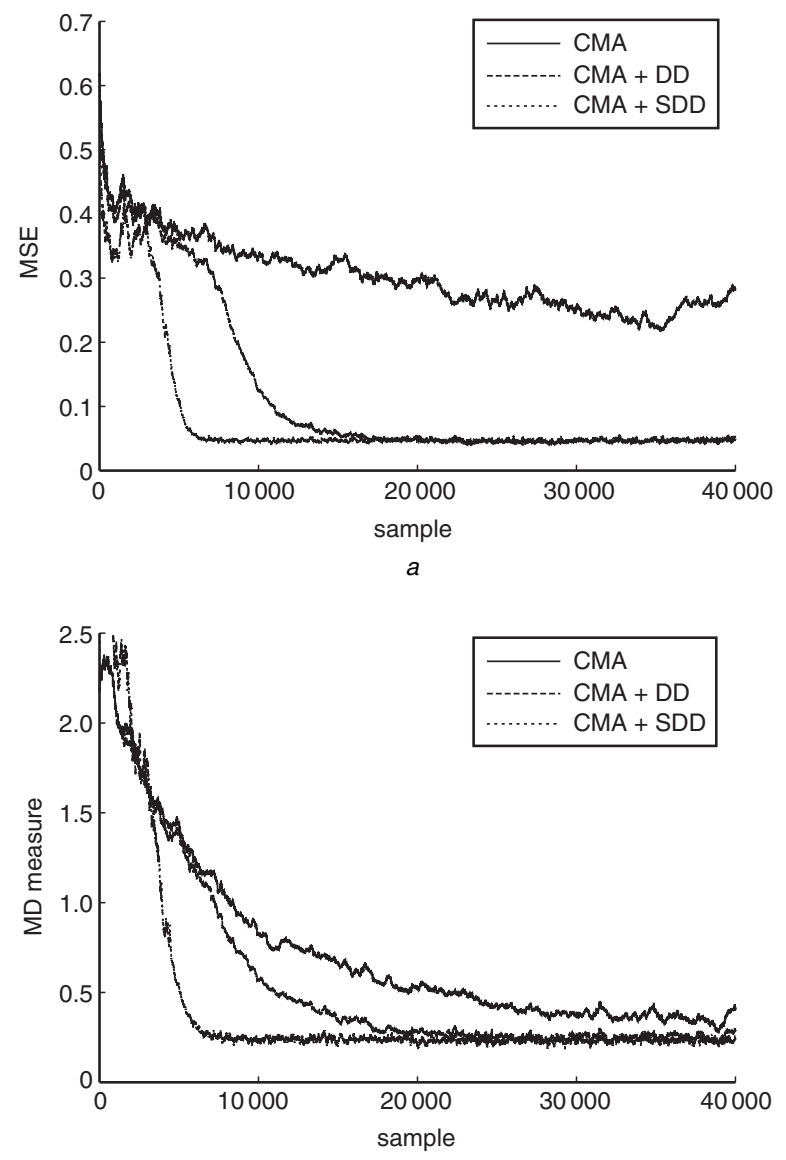

$b$

Fig. 2 Comparison of convergence performance in terms of estimated MSE and MD measure for example 1

$a$ Estimated MSE

$b$ MD measure

together with the length of data samples $N_{\text {MSE }}$ for estimating the MSE at each weight adaptation.

Example 1: In this example, 16-QAM symbols were transmitted through the 22-tap channel, whose CIR is listed in Table 2. The noise power was set to $\sigma_{e}^{2}=$ 0.0157, giving rise to a channel signal to noise ratio (SNR) of $25 \mathrm{~dB}$. The learning curves of the three blind equalisers, the CMA, the $\mathrm{CMA}+\mathrm{DD}$ and the $\mathrm{CMA}+\mathrm{SDD}$, are compared in Fig. 2. The equaliser output signal constellations after convergence are shown in Fig. 3. It can be seen that both the concurrent CMA and SDD scheme and the concurrent CMA and DD scheme have the same steady-state performance, but the former has a faster convergence speed for this example.

Example 2: In this example, the transmitted data symbols were 64-QAM and the channel given in Table 2 was used. With a noise power of $\sigma_{e}^{2}=0.00209$, the SNR was $40 \mathrm{~dB}$. The learning curves of the three blind equalisers, in terms of the estimated MSE and MD measure, are depicted in Figs. $4 a$ and $4 b$, respectively. The equaliser output signal constellations after convergence are shown in Fig. 5. It can be seen that for this example the concurrent CMA and SDD scheme has a faster convergence rate than the concurrent CMA and DD scheme, and both blind equalisers have similar steady-state equalisation performance.

Example 3: The data constellation was 256-QAM and the 22-tap channel given in Table 2 was used in the simulation. The noise power was set to $\sigma_{e}^{2}=8.4 \times 10^{-7}$, giving rise to an SNR of $80 \mathrm{~dB}$. The learning curves of the three blind
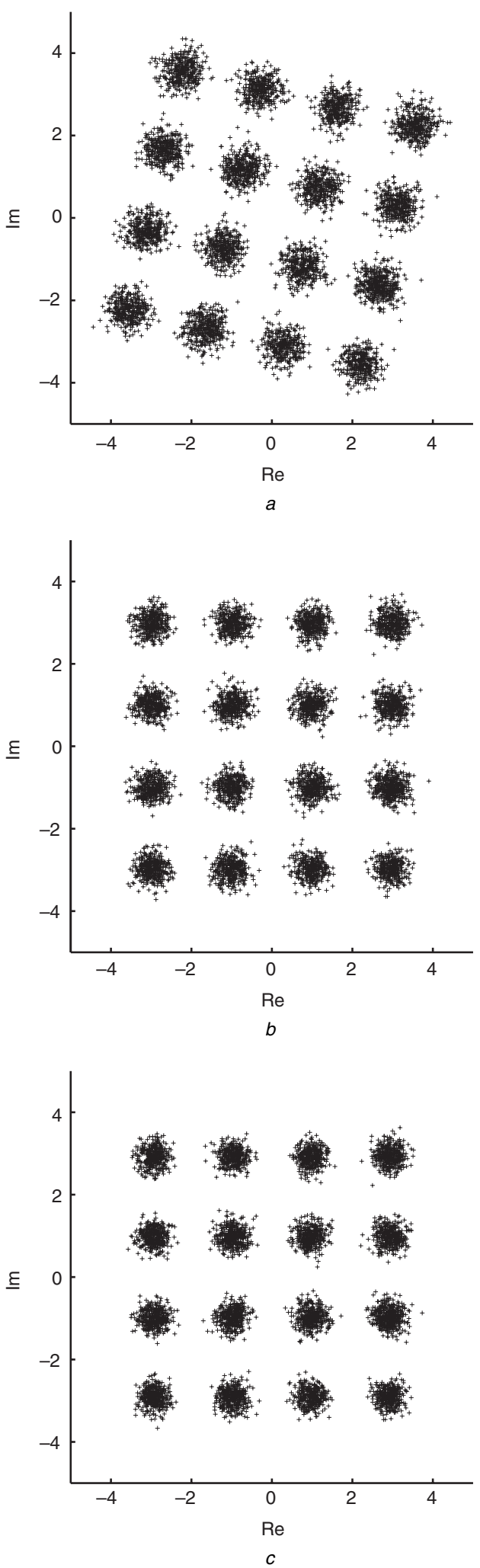

Fig. 3 Equaliser output signal constellations after convergence for example 1

$a$ CMA

$b \mathrm{CMA}+\mathrm{DD}$

$c \mathrm{CMA}+\mathrm{SDD}$

equalisers, in terms of the estimated MSE and MD measure, are depicted in Figs. $6 a$ and $6 b$, respectively. The equaliser output signal constellations after convergence are illustrated in Fig. 7. For this example, it is seen that the proposed new scheme converged marginally faster than the concurrent CMA and DD scheme, and both the concurrent blind equalisers have the same steady-state equalisation performance. 

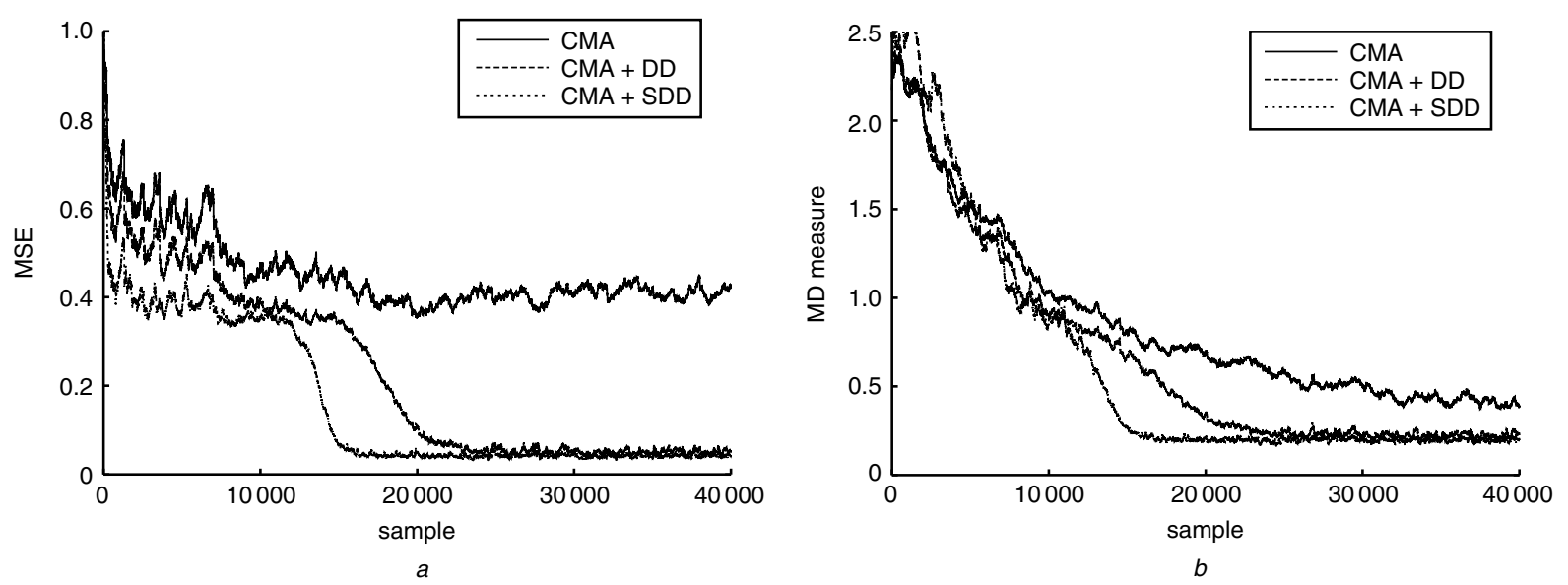

Fig. 4 Comparison of convergence performance in terms of estimated MSE and MD measure for example 2 $a$ Estimated MSE $b$ MD measure
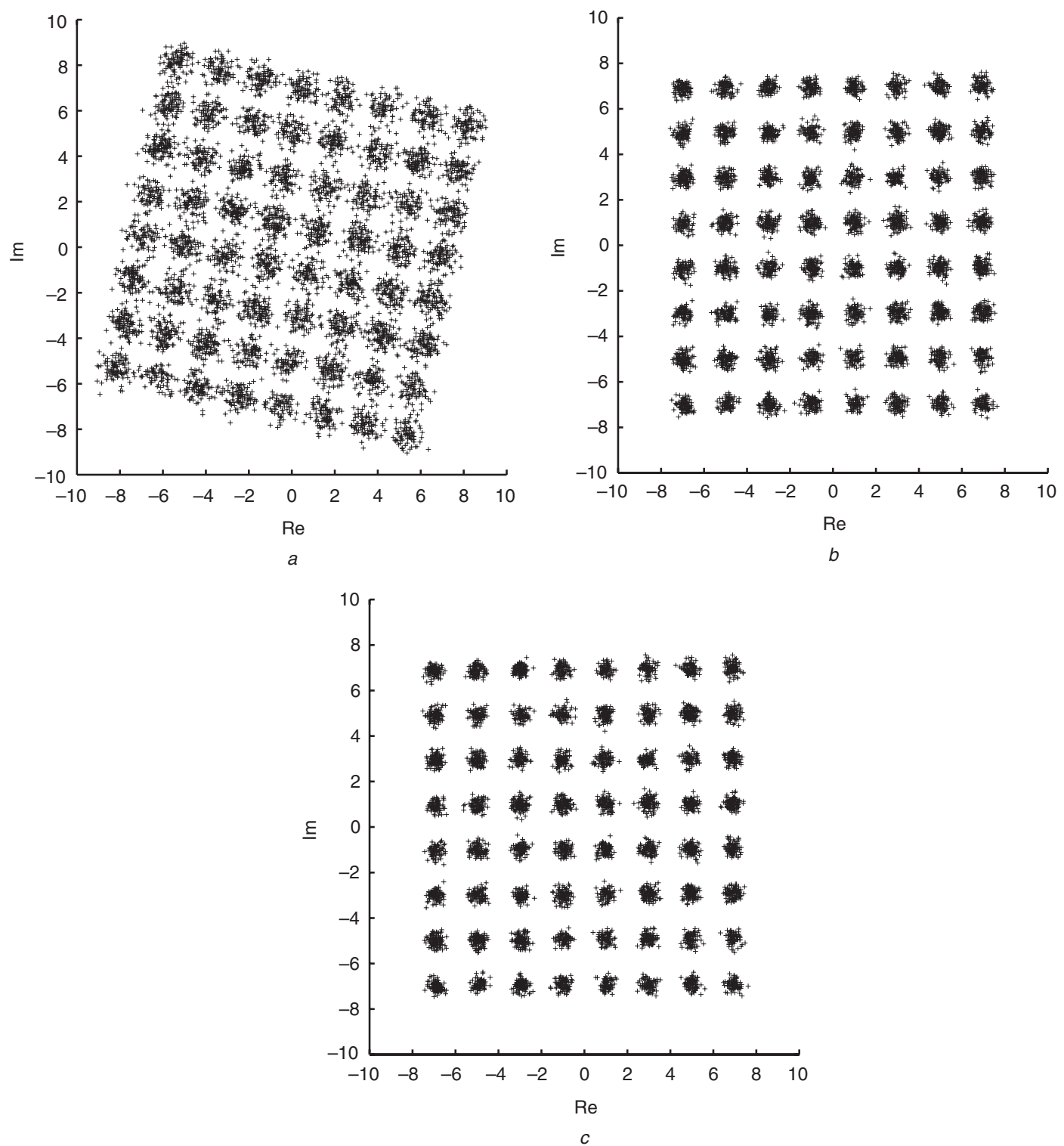

Fig. 5 Equaliser output signal constellations after convergence for example 2

$a$ CMA

$b$ CMA + DD

$c \mathrm{CMA}+\mathrm{SDD}$ 


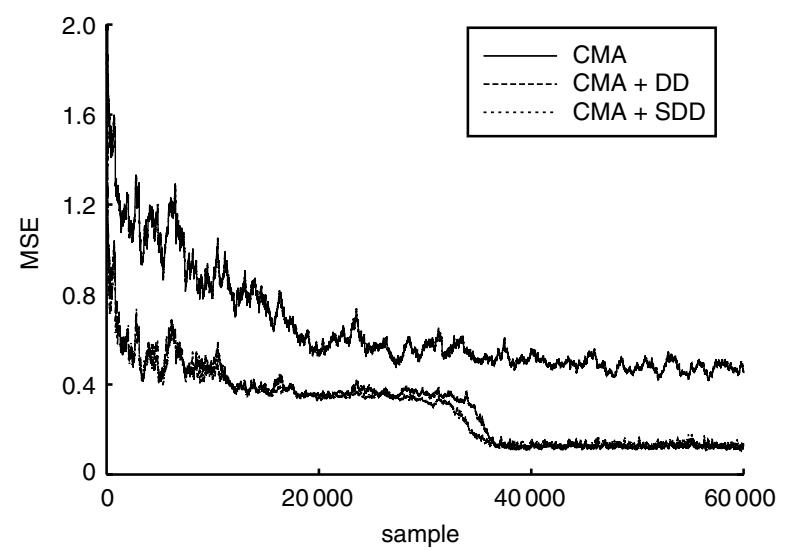

a

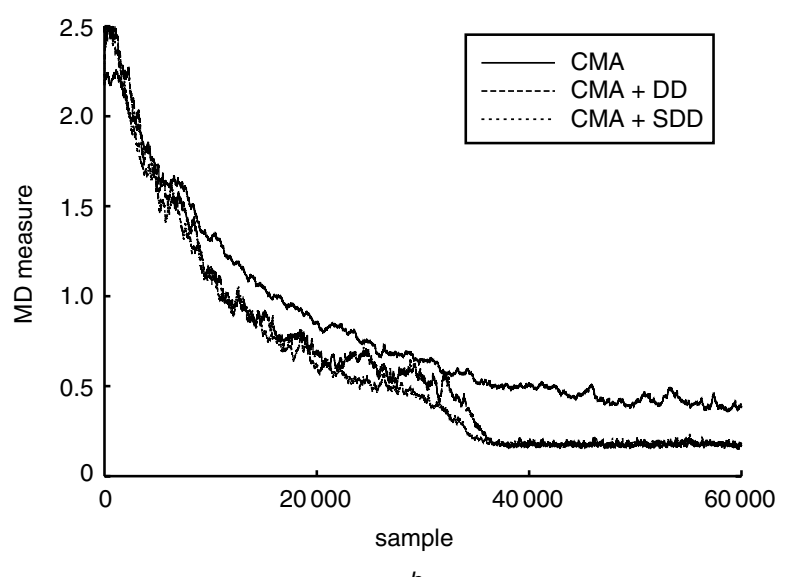

b

Fig. 6 Comparison of convergence performance in terms of estimated MSE and MD measure for example 3 a Estimated MSE $b$ MD measure
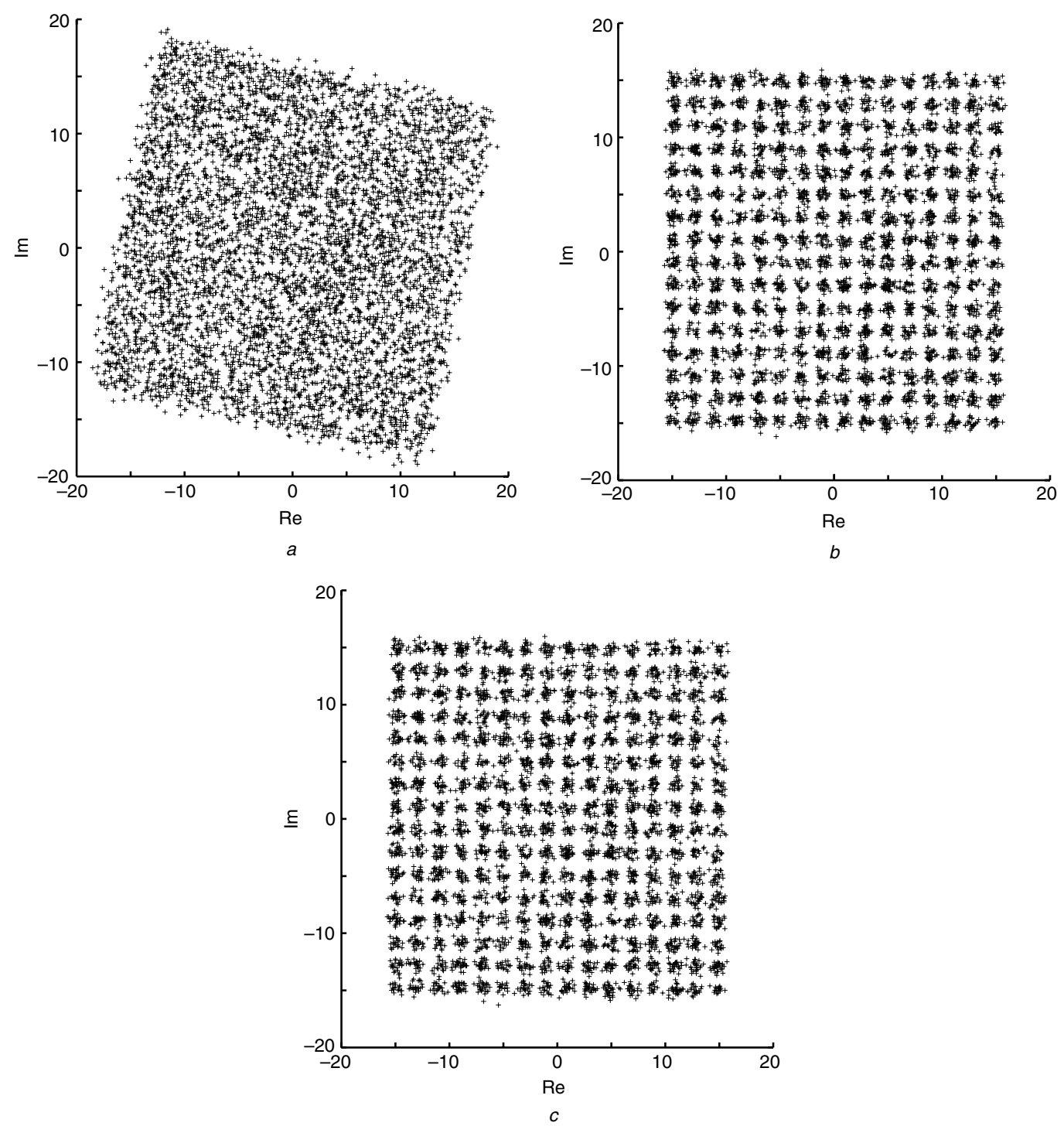

Fig. 7 Equaliser output signal constellations after convergence for example 3

$a$ CMA

$b$ CMA + DD

$c \mathrm{CMA}+\mathrm{SDD}$

Example 4: The data constellation was again 256-QAM but the 5-tap channel given in Table 3 was used in the simulation. With a noise power of $\sigma_{e}^{2}=1.43 \times 10^{-7}$, the SNR was $90 \mathrm{~dB}$. The learning curves of the three blind equalisers, in terms of the estimated MSE and MD measure, are depicted in Figs. $8 a$ and $8 b$, respectively, while the equaliser output signal constellations after convergence are illustrated in Fig. 9. For this example, the faster convergence 

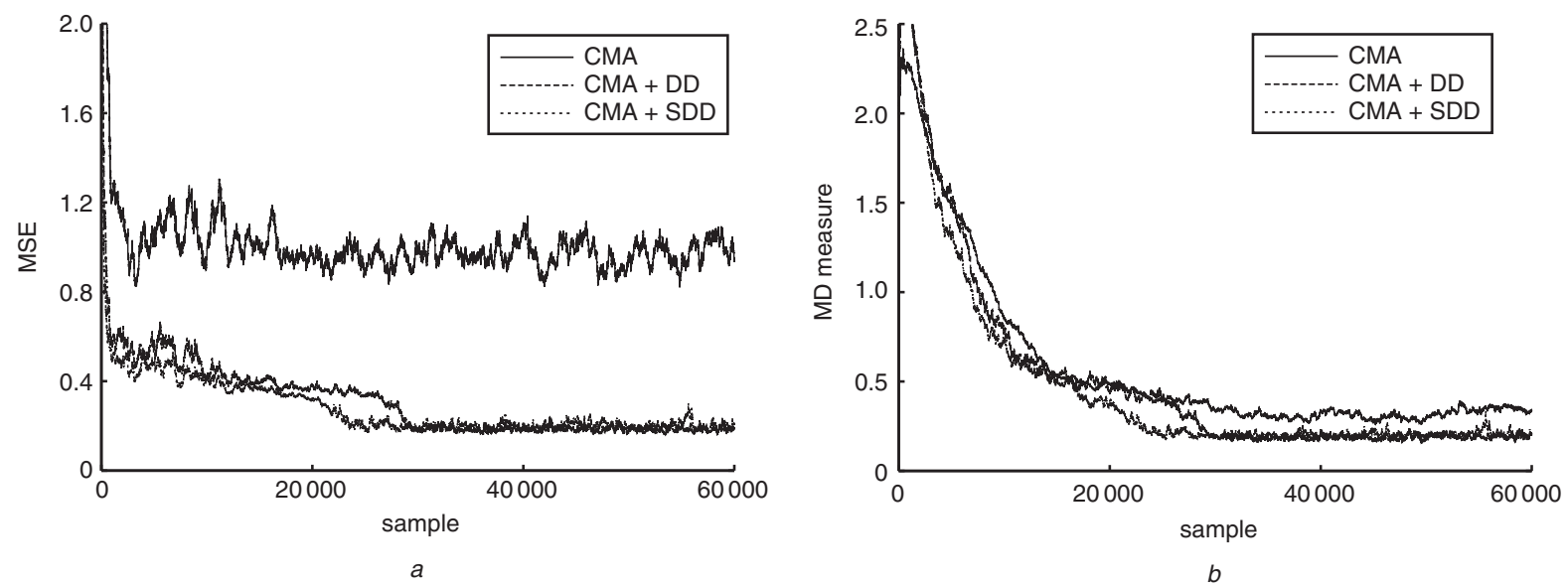

Fig. 8 Comparison of convergence performance in terms of estimated MSE and MD measure for example 4 $a$ Estimated MSE $b$ MD measure
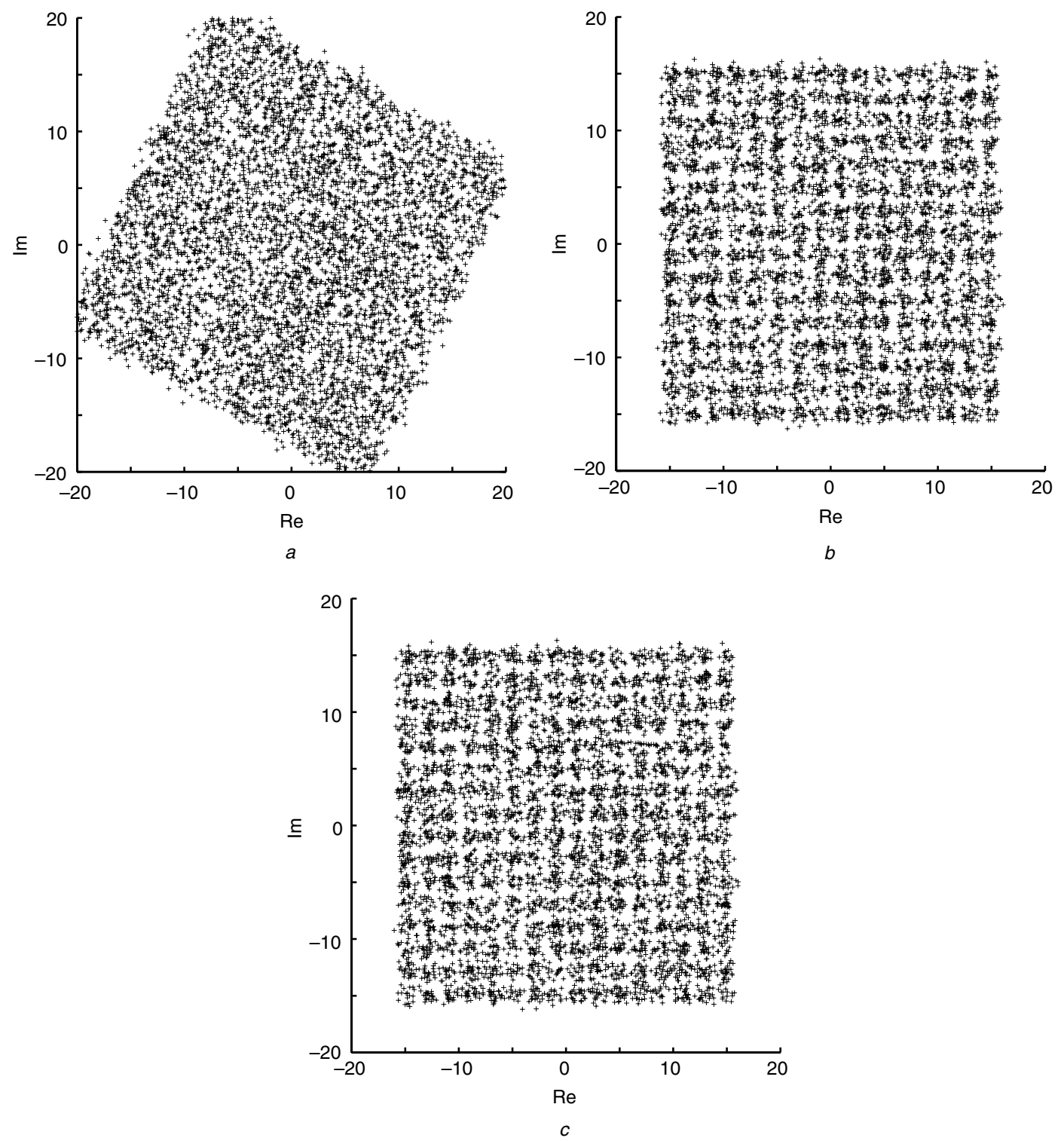

Fig. 9 Equaliser output signal constellations after convergence for example 4 a CMA

$b$ CMA + DD

$c \mathrm{CMA}+\mathrm{SDD}$ 
speed of the proposed new scheme over the concurrent CMA and DD scheme can clearly be seen. Again, both the concurrent blind equalisers are seen to have the same steady-state performance.

\section{Conclusions}

In this paper, a novel low-complexity blind equalisation scheme has been proposed based on operating a CMA equaliser and an SDD equaliser concurrently. Compared with a state-of-the-art low-complexity blind equalisation scheme, namely the recently introduced concurrent CMA and DD blind equaliser, the proposed concurrent CMA and SDD blind equaliser has simpler computational requirements, faster convergence rate and identical steady-state equalisation performance. This new blind equaliser, together with the concurrent CMA and DD blind equaliser, offer practical alternatives to blind equalisation of higher-order QAM channels and provide significant equalisation improvement over the standard CMA based blind equaliser.

\section{References}

1 Godard, D.: 'Self-recovering equalisation and carrier tracking in two-dimensional data communication systems', IEEE Trans. Commun., 1980, 28, pp. $1867-1875$

2 Treichler, J.R., and Agee, B.G.: 'A new approach to multipath correction of constant modulus signals', IEEE Trans. Acoust. Speech Signal Process., 1983, 31, (2), pp. 459-472

3 Treichler, J.R.: 'Application of blind equalisation techniques to voiceband and RF modems'. Preprints 4th IFAC Int. Symp. on Adaptive systems in control and signal processing, France, 1992, pp. $705-713$
4 Jablon, N.K.: 'Joint blind equalization, carrier recovery and timing recovery for high-order QAM signal constellations', IEEE Trans. Signal Process., 1992, 40, (6), pp. 1383-1398

5 Johnson, R., Jr., Schniter, P., Endres, T.J., Behm, J.D., Brown, D.R., and Casas, R.A.: 'Blind equalization using the constant modulus criterion: a review', Proc. IEEE, 1998, 86, (10), pp. 1927-1950

6 Macchi, O., and Eweda, E.: 'Convergence analysis of self-adaptive equalizers', IEEE Trans. Inf. Theory, 1984, 3, (2), pp. 161-176

7 De Castro, F.C.C., De Castro, M.C.F., and Arantes, D.S.: 'Concurrent blind deconvolution for channel equalization'. Proc. ICC'2001, Helsinki, Finland, 11-15 June 2001, vol. 2, pp. 366-371

8 Benveniste, A., and Goursat, M.: 'Blind equalisers', IEEE Trans. Commun., 1984, 32, pp. 871-883

9 Bellini, S., and Rocca, F.: 'Blind deconvolution: polyspectra or Bussgang techniques?', in Biglieri, E., and Prati, G. (Eds.): 'Digital communications' (North Holland, Amsterdam, 1986), pp. 251-263

10 Picchi, G., and Prati, G.: 'Blind equalization and carrier recovering using a 'stop-and-go' decision directed algorithm', IEEE Trans. Commun., 1987, 35, pp. 877-887

11 Haykin, S. (Ed.): 'Blind deconvolution' (Prentice Hall, Englewood Cliffs, NJ, 1994)

12 Chen, S., McLaughlin, S., Grant, P.M., and Mulgrew, B.: 'Reducedcomplexity multi-stage blind clustering equaliser'. Proc. ICC'93, Geneva, Switzerland, 1993, vol. 2, pp. 1149-1153

13 Chen, S., McLaughlin, S., Grant, P.M., and Mulgrew, B.: 'Multi-stage blind clustering equaliser', IEEE Trans. Commun., 1995, 43, (3), pp. $701-705$

14 Karaoguz, J., and Ardalan, S.H.: 'A soft decision-directed blind equalization algorithm applied to equalization of mobile communication channels'. Proc. ICC'92, Chicago, USA, 1992, vol. 3 pp. $343.4 .1-343.4 .5$

15 Chen, S., Cook, T.B., and Anderson, L.C.: 'Blind FIR equalisation for high-order QAM signalling'. Proc. 6th Int Conf. on Signal processing', Beijing, China, 26-30 Aug. 2002, pp. 1299-1302

16 Chen, S., Cook, T.B., and Anderson, L.C.: 'A comparative study of two blind FIR equalisers', Digit. Signal Process., 2004, to be published

17 Anfinsen, S.N., Herrmann, F., and Nandi, A.K.: 'Blind signal equalisation', in Nandi, A.K. (Ed.): 'Blind estimation using higherorder statistics' (Kluwer, Boston, MA, USA, 1999), pp. 27-101

18 Bateman, S.C., and Ameen, S.Y.: 'Comparison of algorithms for use in adaptive adjustment of digital data receivers', IEE Proc. I, Commun. Speech Vis., 1990, 137, (2), pp. 85-96 